\title{
PRIMERAS EVIDENCIAS DE INTERACCIÓN INSECTO-PLANTA EN EL NEÓGENO DEL NOROESTE DE LA ARGENTINA
}

\author{
MARICEL YANINA HORN \\ Secretaría General de Ciencia y Técnica, Universidad Nacional del Nordeste, Centro de Ecología Aplicada del Litoral, \\ Ruta 5, km 2,5 3400, Corrientes, Argentina. yaninahorn@ hotmail.com \\ KAREN ADAMI RODRIGUES \\ Centro Multidiciplinar, Universidade Federal do Acre, Campus Floresta, 69980-000, Cruzeiro do Sul, AC, Brasil. \\ karen.adami@gmail.com

\section{LUISAMATILDEANZÓTEGUI} \\ Facultad de Ciencias Exactas, Naturales y Agrimensura, Universidad Nacional del Nordeste, Av. Libertad, 5460, 3400 \\ Corrientes, Argentina. luisaanzotegui@gmail.com
}

\begin{abstract}
This study provides the first evidence for plantinsect associations from fossil leaf impressions of the San José and Palo Pintado formations, dated as middle and upper Miocene age, from northwestern Argentina. The size, shape and arrangement of leaf damage on several plant hosts consists of various chew marks made by mandibulate insects occurring along the leaf blades of Malvaciphyllum quenquiadensis Anzótegui and Cristalli (Malvaceae), hole feeding along the leaf edge of Nectandra saltensis Anzótegui (Lauraceae), mines within the internal tissues of $M$. quenquiadensis, and oviposition scars in Cedrela sp. (Meliaceae). Based on specific, identifiable features of the plant damage, the likely producers responsible for this damage include external foliage feeders such as Orthoptera, Phasmoptera and Coleoptera; leaf miners from the Lepidoptera but possibly Hymenoptera and Diptera; and ovipositing insects representing the Odonata. Many of these insect groups were previously known from earlier Paleogene deposits of Argentina, and similar ovipositional damage has been documented from the early Eocene of Rio Negro and middle Eocene of Chubut in Patagonia, indicating geochronological continuity and occurrence in marsh and open woodland plant communities under warm and seasonal climatic conditions.
\end{abstract}

Key words: insect-plant interaction, Neogene, northwestern Argentina.

\section{INTRODUCCIÓN}

El objetivo de esta contribución es dar a conocer las primeras evidencias de interacción insecto-planta halladas en impresiones de Angiospermas (Magnoliophyta), de Malvaciphyllum quenquiadensis Anzótegui (Malvaceae), de la Formación San José, y Nectandra saltensis Anzótegui (Lauraceae), Sapium sp. (Euphorbiaceae) y Cedrela sp. (Meliaceae), de la Formación Palo Pintado, ambas del Mioceno del Valle Calchaquí. Por la novedad que representan se considera que el análisis de estos ejemplares brindará un nuevo aporte al conocimiento paleontológico, debido a que son los únicos hallazgos en el Neógeno de la República Argentina. Existen como antecedentes en este país los siguientes trabajos de Patagonia, procedentes de las paleofloras de Laguna del Hunco (Eoceno temprano, provincia de Chubut) y de Río Pichileufú (Eoceno medio, provincia de Río Negro): de la primera, Wilf et al. (2005) dan a conocer 52 tipos diferentes de daños a los que agruparon en cuatro grupos funcionales de herbivoría; de la segunda, Sarzetti et al. (2008) hallaron trazas producidas por abejas de la familia Megachilidae (Hymenoptera), y Sarzetti \& Genise (2009) describen diez morfotipos de agallas, reconociendo cinco ichnotaxones de minaciones. Por último en ambas paleofloras Sarzetti et al. (2009) describen oviposiciones endofíticas de Odonatos en compresiones/impresiones de hojas. No obstante, el análisis de las interacciones insecto-planta fósiles es un campo poco explorado en nivel mundial, debido a que es un tema relativamente nuevo en la Paleontología, el que recién comenzó a recibir más atención de parte de la comunidad científica en las últimas décadas del siglo pasado. Entre otros merecen citarse los aportes de Sharov (1966), Southwood (1973), Vialov (1975), Scott et al. (1985, 1992, 2004), Rozefelds \& Sobbe (1985), Labandeira \& Bell (1990), Stephenson \& Scott (1992), Smith (1994), Beck \& Labandeira (1998), Howell et al. (1998), Wilf \& Labandeira (1999), Gullan \& Cranston (2000), Labandeira (2002, 2007), Adami-Rodrigues (2003), Adami-Rodrigues et al. (2004a,b), Wilf et al. (2005) y Taylor et al. (2009).

Los registros paleontológicos demuestran que las interacciones insecto-planta se establecieron ya entre los primeros habitantes terrestres, sufriendo transformaciones adaptativas que derivaron en muchos casos en evolución conjunta. Estas relaciones se establecieron por la necesidad de nutrición, refugio y reproducción tanto de plantas como de los insectos.

Se estima que actualmente la mitad de las especies de insectos presenta algún grado de dependencia con las plantas, siendo la alimentación (herbivoría) o procesos relacionados a ella, una de las principales relaciones conocidas, dado que los vegetales representan una fuente segura y accesible de alimento. 
En el registro fósil la evidencia de interacción o asociación de insectos herbívoros queda expuesta por los daños ocasionados a las plantas, que se reconocen por la reacción necrótica del tejido en vida, alrededor del sitio dañado. Estos daños o marcas han sido estudiados, nominados y clasificados por su morfología. Así, Vialov (1975) propuso una clasificación formal para los daños de las plantas, que se incluyen en el Grupo Phagophytichnidea, el que a su vez fue dividido en dos Subgrupos Phagolignichnida (daños en leños y corteza) y Phagophytichnida (estructuras sobre hojas y tallos que no son propias de la actividad de las plantas) con las siguientes familias: Phagophytichnidae (escisiones en el tejido de las hojas), Paleominidae (minas en tejidos de hojas), Paleogallidae (agallas) y Paleoovoididae ("huevos" en hojas y otros órganos). Por otra parte, Labandeira (2002) reunió los daños por herbivoría en distintos grupos funcionales alimenticios, que reflejan las estrategias de insectos fitófagos, las que en su mayoría también se observan en sus parientes actuales. En su tercera versión Labandeira et al. (2007), presentan una guía en la que exponen 150 tipos diferentes de daños según sus características morfológicas (entre otros, tamaño, forma y disposición en los tejidos de las plantas). La cuarta versión (Labandeira, com. pers. 2010) incluirá además de 90 tipos de daños nuevos, datos adicionales como claves dicotómicas e ilustraciones de los análogos modernos.

Artrópodos detritívoros se conocen desde el Silúrico, pero desde el Carbonífero se reconoce a la herbivoría como el tipo alimenticio principal (Scott et al., 1992). No obstante, la evidencia fósil sugiere que la mayor diversidad de insectos fitófagos comienza después del Cretácico (Gullan \& Cranston, 2000), coincidiendo con la diversificación de las angiospermas. En esta época también se observan diversas adaptaciones y nuevas conductas de los insectos en respuesta a las estrategias químicas, físicas y morfológicas de las plantas para desalentar la herbivoría.

Las plantas, en ocasiones, también actúan como sitios donde los insectos depositan sus huevos (oviposición). Entre los actuales se distingue entre oviposición endofítica y exofítica. La oviposición exofítica está representada por la agregación de huevos bajo un mucus que los cubre y que puede preservarse durante la fosilización, mientras que la oviposición endofítica está representada por huevos solitarios dentro de los tejidos de la planta depositados por animales con ovipositor (Vasilenko, 2005). La evidencia de oviposición en los fósiles, se reconoce externamente por la presencia de cicatrices en las hojas debido a la reacción del tejido en respuesta al estímulo, y/o por los daños que producen las estructuras que conforman el ovipositor, del cuál también pueden hallarse algunas partes sueltas (Labandeira, 2002). Cicatrices lenticulares causadas por la inserción del ovipositor de algunos insectos (cicatrices oviposicionales) han sido descriptas, por ejemplo, en tallos de Calamites y Equisetites (Pensilvaniano) y en hojas de Schmeissneria (Ginkgophyta), para el Mesozoico de Europa y América del Norte (Taylor et al., 2009).

\section{MATERIAL Y MÉTODOS}

Se sigue la publicación Guide to insect and other damage types on compressed plant fossils propuesta por Labandeira et al. (2007) para el reconocimiento e identificación de los tipos de daños, y como consecuencia se utiliza la sigla DT (damage type), para hacer referencia a los mismos. Los ejemplares fueron observados con una lupa binocular

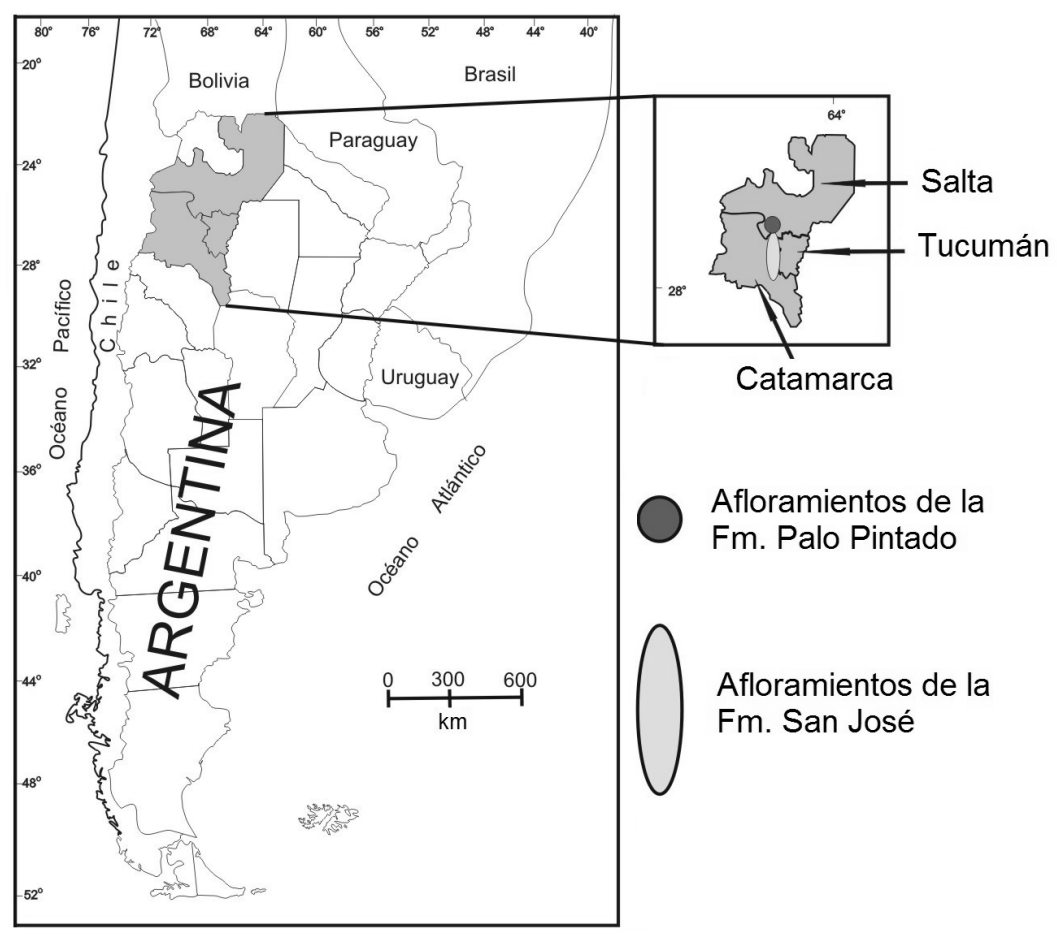

Figura 1. Mapa de ubicación de las areas de estudio.

Figure 1. Location map of the study areas. 
Olympus 5251, y fotografiados con una cámara digital Olympus SP 350; pertenecen a la Colección CTES-PB (Corrientes-Paleobotánica), de la Facultad de Ciencias Exactas y Naturales y Agrimensura de la Universidad Nacional del Nordeste, Argentina.

\section{MARCO GEOLÓGICO Y PALEOCOMUIDADES}

La Formación San José integra la unidad basal del Grupo Santa María y aflora en las provincias de Catamarca y Tucumán (Figura 1). Las impresiones provienen del arroyo La Quenquiada, ubicado al este de la localidad Amaicha del Valle, en el noroeste de la provincia de Tucumán. Las mismas se encuentran en una secuencia sedimentaria de más o menos 4-5 m, compuesta por pelitas y margas verde-amarillentas, estratificadas en capas muy delgadas, con intercalaciones de areniscas finas de neto origen lacustre (Anzótegui \& Cristalli, 2000). La Formación Palo Pintado se encuentra al Sur de la provincia de Salta, integra el Grupo Payogastilla y se halla intercalada entre las formaciones Angastaco y San Felipe (Figura 1). Litológicamente está compuesta por areniscas y frecuentes pelitas, mientras que los conglomerados son subordinados. El conjunto muestra una disposición en ciclos grano decrecientes (Anzótegui, 1998, 2006). Las impresiones provienen de la parte basal de la Formación, de las localidades Quebrada de Alfredo y Quebrada El Estanque.

Las hojas fósiles con daños (Cedrela sp., Sapium sp., Nectandra saltensis y Malvaciphyllum quenquiadensis) de las formaciones Palo Pintado y San José (Mioceno), provienen de vegetales arbóreos y arbustivos, que se habrían desarrollado bajo un clima cálido y húmedo con estacionalidad breve, integrando bosques de ribera (Anzótegui et al., 2009).

\section{RESULTADOS Y DISCUSIÓN}

El estudio de las impresiones dio por resultado la identificación de varias lesiones en las hojas, producidas por diferentes actividades de los insectos. Se hallaron tres tipos de daños por herbivoría (marcas mandibulares, perforaciones y excavaciones en los tejidos) y otro tipo que representa cicatrices por oviposición. Algunos de los ejemplares presentan más de un tipo de estos daños, excepto las cicatrices por oviposición que se encontraron en un solo ejemplar.

\section{Marcas mandibulares}

En un fragmento de hoja de Malvaciphyllum quenquiadensis (CTES-PB: 12471a,b) se observan dos escisiones, una en el margen superior y otra en el lateral; la primera es de $3 \mathrm{~mm}$ de largo por $6 \mathrm{~mm}$ de profundidad, y la segunda de $4 \mathrm{~mm}$ de largo por $2 \mathrm{~mm}$ de profundidad, ambas limitadas por un reborde más grueso y oscuro (Figura 2A). Estas lesiones se relacionan los tipos DT 12 y DT 15 (margin feeding); con el primero por la forma semicircular y el ángulo de $180^{\circ}$ del arco, y con el segundo por la profundidad de la escisión que se aproxima a la vena media.

\section{Perforaciones}

En Nectandra saltensis (CTES-PB: 6653) las perforaciones son circulares y solitarias, miden $3 \mathrm{~mm}$ de largo por $2,5 \mathrm{~mm}$ de ancho. Se relacionan por su forma y tamaño (menos de $1 \mathrm{~mm}$ a $5 \mathrm{~mm}$ de diámetro) con las perforaciones circulares que se ilustran en los tipos DT 01 y DT 02 (hole feeding). En Sapium sp. (CTES-PB: 10779a,b, 10780, 10792, 10794) las perforaciones son oval-alargadas, se ubican paralelas a la vena media, presentan bordes irregulares y las dimensiones se encuentran entre los rangos de $1 \mathrm{~mm}$ a $6 \mathrm{~mm}$ de largo por $1 \mathrm{~mm}$ de ancho. Se las relaciona con los tipo DT 07 y DT 08 (hole feeding), por la forma elongada recta a curvilínea, la ubicación paralela a la vena media y por los bordes irregulares (Figuras 2E-F).

\section{Excavaciones en los tejidos}

En Nectandra saltensis (CTES-PB: 6653) se observa un surco con una porción filiforme serpenteante que comienza su recorrido aparentemente sobre la vena media y termina en una porción engrosada de $10 \mathrm{~mm}$ de largo por $1 \mathrm{~mm}$ de ancho, con márgenes definidos, de dirección casi rectilínea y paralela a la vena media (Figura 2B). Por estas características se relaciona con el tipo DT 41 (mining), por el recorrido largo, cuyo inicio es filiforme y espiralado, ensanchándose hacia el final. En Malvaciphyllum quenquiadensis (CTES-PB: 12471a,b) se observan dos surcos de 4-6 mm de largo por 1 $\mathrm{mm}$ de ancho cada uno (Figura 2A), los que parten de una de las venas primarias y en su recorrido abarcan los espacios intercostales describiendo leves curvaturas hacia la base de la hoja (Figura 2G). Estos se relacionan con el tipo DT 105 (mining), por la forma, el ancho, la ubicación entre dos venas secundarias, márgenes débiles y la ausencia de partículas de excremento.

\section{Cicatrices de oviposición}

En Cedrela sp. (CTES-PB: 8028a,b) se hallaron 11 marcas individuales ubicadas en hilera limitando a la vena media; son ovadas con la base redondeada, miden $1 \mathrm{~mm}$ de largo por $0,5 \mathrm{~mm}$ de ancho, tienen bordes bien marcados y están separadas entre sí por más menos $3 \mathrm{~mm}$ de distancia (Figuras 2H,I). Por estas características se relacionarían con el DT 175 (Labandeira, com. pers. 2010). Sin embargo por la disposición alineada y próxima a la vena central, por la cantidad, forma, tamaño y distancia existente entre ellas, se asemejan a las de Paleoovoidus rectus Vasilenko, 2005 (in Sarzetti et al., 2009, figuras 2.3 y 2.4 ).

\section{Grupos alimenticios para insectos herbívoros identificados a partir de las lesiones}

Entre los nueve grupos alimenticios funcionales para insectos herbívoros sobre tejido vivo se reconocen aquí por los tipos de daños antes descriptos, dos grupos (Labandeira, 2002): (A) grupo de consumidores de follaje externo, con dos subtipos, (i) alimentación marginal, caracterizada por escisiones (mordisqueo, Beck \& Labandeira, 1998) u otro tipo de remoción del margen de las hojas (continua, discontinua y apical), y (ii) marcas en forma de agujeros circulares o poligonales en los que las porciones internas de 

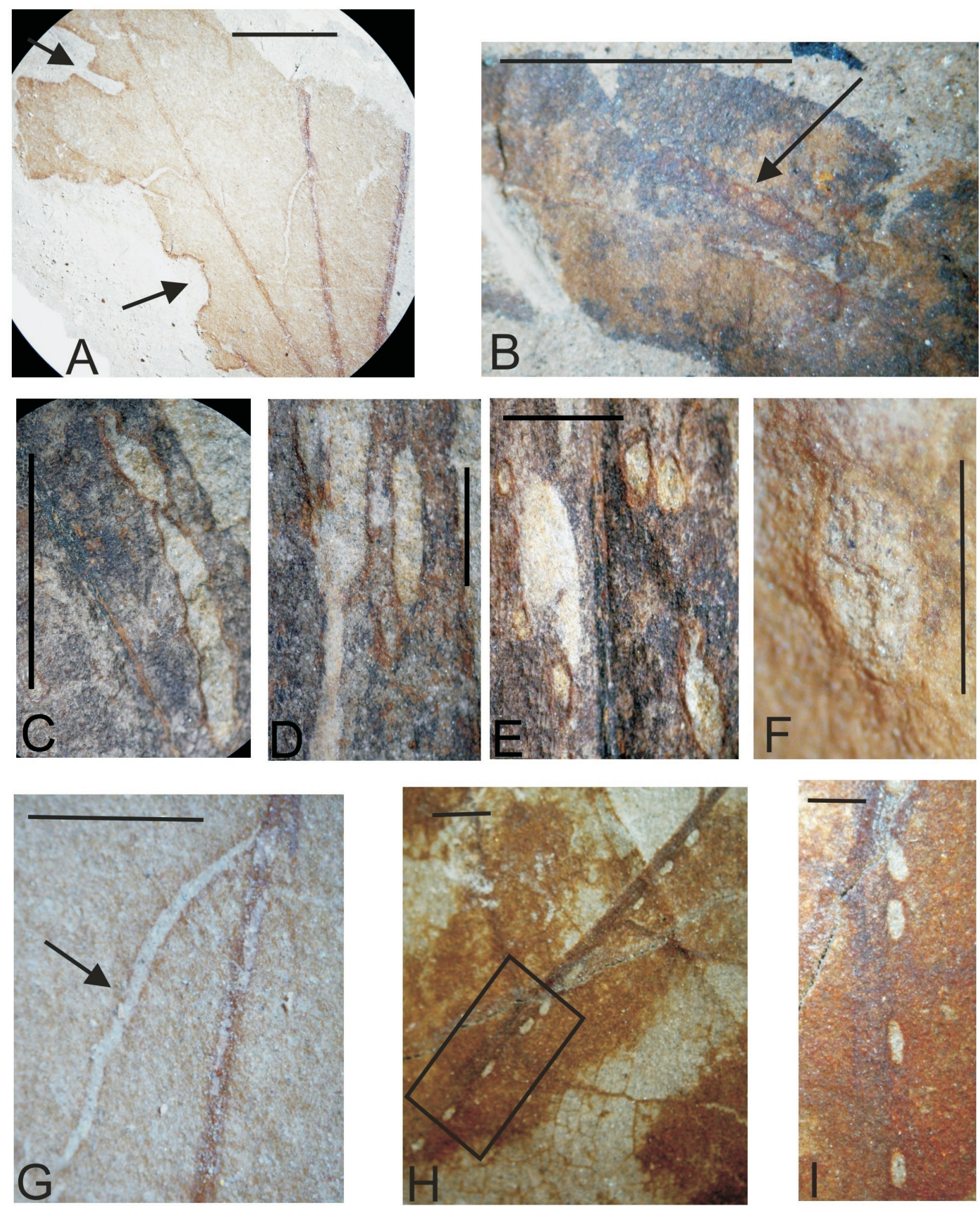

Figura 2. A, Alimentación marginal (DT 12 y DT 15 en Labandeira et al., 2007) y minas en Malvaciphyllum quenquiadensis Anzótegui y Cristalli, CTES PB: 12471 a/b; B, mina (DT 41 en Labandeira et al., 2007) en Nectandra saltensis CTES PB: 6653; C-E, agujero de alimentación (DT 07 y DT 08 en Labandeira et al., 2007) en Sapium sp., CTES PB: 10780; F, agujero de alimentación (DT 02 en Labandeira et al., 2007) en Nectandra saltensis Anzótegui, CTES PB: 6653; G, detalle de una mina en Malvaciphyllum quenquiadensis (DT 105 en Labandeira et al., 2007), CTES PB: 12471 a/b; H, cicatrices de oviposición (DT175; Labandeira coms. pers., 2010) en Cedrela sp., CTES PB: $8028 \mathrm{a} / \mathrm{b}$; I, detalle del área seleccionada en H. Escalas: $\mathrm{A}=6 \mathrm{~mm} ; \mathrm{B}=10 \mathrm{~mm}$; C-D $=6 \mathrm{~mm} ; \mathrm{E}=4 \mathrm{~mm} ; \mathrm{F}-\mathrm{I}=3 \mathrm{~mm}$.

Figure 2. A, Margin feeding (DT 12 and DT 15 of Labandeira et al.,2007) and mines in Malvaciphyllum quenquiadensis Anzótegui y Cristalli, CTES PB: 12471 a/b; B, mine (DT 41 of Labandeira et al., 2007) in Malvaciphyllum quenquiadensis CTES PB: 12471 a/b; C-E, hole feeding (DT 07 and DT 08 of Labandeira et al., 2007) in Sapium sp., CTES PB: 10780; F, hole-feeding (DT 02 of Labandeira et al., 2007) in Nectandra saltensis Anzótegui, CTES PB: 6653; G, mine detail in Malvaciphyllum quenquiadensis (DT 105 of Labandeira et al., 2007), CTES PB: 12471 a/b; H, oviposition scars (DT175; Labandeira pers. comm., 2010) in Cedrela sp., CTES PB: 8028 a/b; I, details of the selected area in $\mathrm{H}$. Scale bars: $A=6 \mathrm{~mm} ; B=10 \mathrm{~mm} ; C-D=6 \mathrm{~mm} ; E=4 \mathrm{~mm} ; F-I=3 \mathrm{~mm}$. 
tejido son removidas; (B) Grupo de reductores foliares, son los consumidores endofíticos que excavan túneles dentro del mesófilo llamados minas; en ellos depositan huevos y se produce el desarrollo larval hasta llegar al estado adulto; según la especialización del insecto pueden ser lineares o tener forma serpenteante y terminar en un extremo ensanchado, que representa la cámara pupal (Scott et al., 1992).

\section{Posibles insectos productores}

La mayoría de los grupos de insectos actuales que se alimentan de follaje externo son estados inmaduros o adultos de casi todas las especies de Orthoptera, Phasmatoptera, Lepidoptera, y un porcentaje menor de Coleoptera e Hymenoptera (Labandeira, 2002). Los minadores comprenden a larvas de cuatro grupos de insectos actuales: Coleoptera, Diptera, Lepidoptera e Hymenoptera. Los órdenes Orthoptera, Hemiptera, Coleoptera, Lepidoptera e Hymenoptera penetran los tejidos de las plantas con sus ovipositores para la inserción de sus huevos en el interior de los mismos.

Respecto a los fósiles, Vasilenko (2005) y Sarzetti et al. (2009), entre otros, atribuyen las oviposiciones del Cenozoico (incluso algunas del Mesozoico), por la forma y tamaño a insectos de las Familias Coenagrionidae y Lestidae (Odonata). Por otra parte, en una lista de insectos fósiles del Cenozoico de Sudamérica Petrulevicius y Martins-Neto (2002) citan para Argentina géneros y especies de los siguientes órdenes: Odonata, Orthoptera, Dermaptera, Hemiptera (subordenes Homoptera y Heteroptera), Coleoptera y Trichoptera, hallados en la Formación Maíz Gordo (Paleoceno inferior) en la provincia de Jujuy, y un género del orden Hymenoptera en la Formación Ventana de la provincia de Río Negro (Paleoceno-Eoceno).

De este estudio se destaca que, entre los órdenes de insectos herbívoros actuales, por el momento Orthoptera, Hymenoptera y Coleoptera, ya fueron citados en el Paleógeno del noroeste de la Argentina por Petrulevicius y MartinsNeto (2002).

Considerando la edad y la región geográfica actual, es posible que especies de estos órdenes también hayan sido las productoras de las trazas aquí descriptas. Por otro lado, queda manifiesta la presencia del Orden Odonata mediante las cicatrices de oviposición en el Eoceno temprano (Río Negro) y medio (Chubut) de Patagonia y el Mioceno Superior de Salta.

Por último, cabe agregar, que las hojas en las que se hallaron los daños en este trabajo, habrían pertenecido a plantas integrantes de bosques de ribera, desarrollados bajo un clima subtropical húmedo (Anzótegui et al., 2009), por lo que se infiere que este habría sido el hábitat en el que vivieron los insectos productores de los daños aquí tratados.

\section{AGRADECIMIENTOS}

Agradecemos a los revisores, particulamente C. Labandeira, por sus valiosas sugerencias. A.S. Gnaedinger y O.F. Galllego, por su apoyo y aporte bibliográfico. Este trabajo fue realizado en el marco de los proyectos PI 96/07, otorgado por la Secretaría General de Ciencia y Técnica de la Universidad Nacional del Nordeste y del PICTO 2007/124 (Agencia/UNNE).

\section{REFERENCIAS}

Adami-Rodrigues, K. 2003. Evidências de interações insecto-planta nos depósitos neopaleozóicos do Rio Grande do Sul. Programa de Pós-Graduação em Geociências, Universidade Federal do Rio Grande do Sul, Tese de Doutoramento, 179 p.

Adami-Rodrigues, K.; Souza, P.A.; Iannuzzi, R. \& Pinto, I.D. 2004a. Hebivoria em floras Gonduânicas do Neopaleózoico do Rio Grande do Sul: análise quantitativa. Revista Brasileira de Paleontologia, 7(2):93-102.

Adami-Rodrigues, K.; Iannuzzi, R. \& Pinto, I.D. 2004b. Permian plant-insect interactions on Gondwana flora from southern Brazil. Fossils and Strata, 51:106-125.

Anzótegui, L.M. 1998. Hojas de angiospermas de la Formación Palo Pintado, Mioceno superior, Salta, Argentina. Parte I: Anacardiaceae, Lauraceae y Moraceae. Ameghiniana, 35(1):25-32.

Anzótegui, L.M. 2006. Paleofloras del Mioceno en los Valles Calchaquíes, Noroeste de Argentina. Facultad de Ciencias Exactas y Naturales y Agrimensura, Universidad Nacional del Nordeste, Tesis Doctoral, 266 p.

Anzótegui, L.M. \& Cristalli, P. 2000. Primer registro de hojas de Malvaceae en el Neógeno de Argentina y Brasil. Ameghiniana, 37(2):169-180.

Anzótegui, L.M.; Horn, M.Y. \& Galli, C. 2009. Nuevos integrantes de la Formación Palo Pintado (Mioceno Superior) del Valle Calchaquí. In: SIMPOSIO ARGENTINO DE PALEOBOTÁNICA Y PALINOLOGÍA, 14, 2009. Resúmenes, Mar del Plata, p. 19.

Beck, A.L. \& Labandeira, C.C. 1998. Early Permian insect folivory on a gigantopterid-dominated riparian flora from north-central Texas. Palaeogeography, Palaeoclimatology, Palaeoecology, 142:139-173.

Gullan, P.J. \& Cranston P.S. 2000. The insects: an outline of Entomology. $2^{\mathrm{a}}$ ed. Blackwell Science, Oxford, 470 p.

Howell, V.D.; Doyen J.T. \& Purcell A.H. 1998. Introduction to insect biology and diversity. $2^{\mathrm{a}}$ ed. Oxford University Press, Oxford, 680 p.

Labandeira, C.C. \& Beall, B.S. 1990. Arthropod terrestriality. In: D.G Mikulic (ed.) Arthropods: short courses in Paleontology, University of Tennessee Press, v. 3, p. 214-256.

Labandeira, C. 2002. The history of associations between plants and animals. In: Herrera, C.M. \& O. Pellmyr (eds.) Plant-animal interactions: an evolutionary approach, Blackwell Science, p. 248-261.

Labandeira, C.C.; Wilf, P.; Johnson, K.R. \& Finnegan M. 2007. Guide to insect (and other) damage types on compressed plant fossils (version 3.0). Available at http://paleobiology.si.edu/ insects/index.html; accessed on 10/10/2009.

Petrulevicius, F. \& Martins-Neto R.G. 2000. Checklist of South American Cenozoic Insects. Acta Geológica Hispánica, 35:135-147.

Rozefelds, A.C. \& Sobbe, I. 1987. Problematic insect leaf mines from the Upper Triassic Ipswich Coal Measures of southeastern Queensland, Australia. Alcheringa, 11:51-57. doi: 10.1080/03115518708618979 
Sarzetti, L.C.; Labandeira, C.C. \& Genise, J.F. 2008. A leafcutter bee trace fossil from the middle Eocene of Patagonia, Argentina, and review of megachilid (Hymenoptera) ichnology. Palaeontology, 51:933-941. doi: 10.1111/j.1475-4983.2008.00787.x

Sarzetti, L.C. \& Genise, J.F. 2009. Agallas y minaciones en hojas del Eoceno temprano y medio de Patagonia y su importancia como indicadores de condiciones paleoambientales. In: REUNIÓN ANUAL DE COMUNICACIONES DE LA ASOCIACIÓN PALEONTOLÓGICA ARGENTINA, 2009. Resúmenes, Buenos Aires, p. 67.

Sarzetti, L.C.; Labandeira, C.C.; Muzón, J.; Wilf, P.; Cúneo, N.R.; Johnson, K.R. \& Genise, J.F. 2009. Odonatan endophytic oviposition from the Eocene of Patagonia: the ichnogenus Paleoovoidus and implications for behavioral stasis. Journal of Paleontology, 83(3):431-447. doi: 10.1666/08-121.1

Scott A.C.; Anderson J.M. \& Anderson H.M. 2004. Evidence of plant-insect interactions in the Upper Triassic Molteno Formation of South Africa. Journal of the Geological Society of London, 161:401-410. doi:10.1144/0016-764903-118

Scott., A.C.; Chaloner, W.G. \& Paterson, S. 1985. Evidence of pteridophyte arthropod interactions in the fossil record. Proceedings of the Royal Society of Edinburgh B, 86:133-140.

Scott, A.C.; Stephenson J. \& Chaloner W.G. 1992. Interaction and coevolution of plants and arthropods during the Paleozoic and Mesozoic. Philosophical Transactions of the Royal Society of London (Biological Sciences), 335:129-165. doi:10.1098/ rstb.1992.0016
Sharov, A.G. 1966. Basic arthropodan stock, with special reference to insects. $1^{\mathrm{a}}$ ed. Oxford, Pergamon Press, $271 \mathrm{p}$.

Smith, D.M.1994. Plant-insect interactions in the fossil record. Phytophaga, 6:35-49.

Stephenson, J. \& Scott, A.C. 1992. The geological history of insectrelated plant damage. Terra Nova, 4:542-552.

Southwood, T.R.E. 1973. The insect/plant relationship an evolutionary perspective. In: H. F. Emden (ed.) Insect/plant relationships, Blackwell Science, p. 3-30.

Taylor, T.N.; Taylor E.L. \& Krings M. 2009. Paleobotany: the biology and evolution of fossil plants. $2^{\mathrm{a}}$ ed. Elsevier, New York, $1230 \mathrm{p}$.

Vialov, O.S. 1975. The fossil traces of nourishment of the insects. Paleontologichesky Sbornik, 1-2:147-155.

Vasilenko, D.V. 2005. Damages on Mesozoic plants from the Transbaikalian locality Chernovskie Kopi. Paleontological Journal, 39(6):54-59.

Wilf, P. \& Labandeira, C.C. 1999. Response of plant-insect associations to Paleocene-Eocene warming. Science, 284:21532156. doi: 10.1126/science.284.5423.2153

Wilf, P.; Labandeira C.C.; Johnson, K.R. \& Cúneo N.R. 2005. Richness of plant insect associations in Eocene Patagonia: a legacy for South American biodiversity. Proceedings of the Nacional Academy of Sciences, U.S.A., 102:2153-2156. doi: 10.1073/pnas.0500516102

Received in December, 2009; accepted in November, 2010. 